\title{
Effect of sting size on the wake of a sphere at subcritical Reynolds numbers
}

\author{
AK Norman*and BJ McKeon ${ }^{\dagger}$ \\ California Institute of Technology, Pasadena, CA 91125
}

\begin{abstract}
Vortex shedding and turbulent motion in the wake of a sphere that is supported using a streamwise-aligned cylindrical sting are investigated at a subcritical Reynolds number of Re $=3800$, using high speed particle image velocimetry. The mechanism by which the presence of a sting of increasing diameter relative to the diameter of the sphere influences the wake, in terms of both the small-scale shear instability and the larger wake instability, is explored and briefly compared with the two-dimensional analog of the splitter plate introduced into a cylinder wake. The difficulties associated with obtaining converged statistics, along with the effect of free stream turbulence and sphere vibrations are detailed. An understanding of the mechanism by which the blockage, or interference, arising from the presence of the sting influences cross-wake communication and downstream development is a necessary precursor to studies of active control of the wake using surface actuation on a sting-mounted sphere.
\end{abstract}

\section{Introduction}

Whereas there have been numerous investigations on the effect of an obstructing, or splitter, plate on the development of the wake behind a cylinder, there has been less study of the weak analog for a threedimensional axisymmetric bluff-body, namely the influence of a cylindrical support sting on the development of the wake behind a sphere. While an interesting problem in its own right, identification of the effect of a finite-sized sting on both the shear layer that is shed from the sphere and the downstream wake is a necessary precursor to detailed studies of active control of the sphere boundary layer (i.e. experiments in which a tether mount is less desirable than a rear sting), with a view to manipulating the magnitude and direction of the resultant forces on the sphere.

For sphere Reynolds numbers (where $R e=\rho U_{\infty} D / \mu$ is based on sphere diameter $D$, free stream velocity $U_{\infty}$, density $\rho$, and dynamic viscosity $\mu$ ) above approximately 800 and below the critical Reynolds number for transition of the sphere boundary layer, $R e \approx 3 \times 10^{5}$, frequencies corresponding to both a small-scale shear layer instability and a larger wake instability are present in the wake, while below this lower limit only the larger scale is observed. ${ }^{1-4}$ With increasing Reynolds number the Strouhal number $\left(S t=f D / U_{\infty}\right.$, where $f$ is the frequency) of the shear instability increases $\operatorname{as}^{2} R e^{n}$, with $0.5 \leq n<1$ for $10^{3}<R e_{D}<10^{5}$, while the large-scale Strouhal number remains approximately equal to 0.2, typical of vortex shedding. In the subcritical regime, computational studies ${ }^{5,6}$ have also found a low frequency motion of the wake, below that of the large-scale vortex shedding frequency.

The ratio of sting diameter to sphere diameter, $\chi=D_{S} / D$ (figure 1), in the cited experimental studies ranges from 0.055 to 0.15 , indicating an unwritten rule of thumb for the size of the sting for minimal interference on the wake. However, Hoerner ${ }^{7}$ investigated the effect of $\chi$ on the drag coefficient and found a negligible effect on the subcritical drag and on the Reynolds number of transition, whereas the drag decreased with increasing $\chi$ in the supercritical regime where the size of the wake is greatly reduced due to the boundary layer becoming turbulent before separation.

Flow over a cylinder has been heavily investigated, and here we review only a few examples of obstructing the wake of a cylinder. Roshko investigated the effect of a splitter plate, placed on the wake centerline and with varying length and distance from the downstream separation point on the cylinder, and found that for

\footnotetext{
*Graduate Student, Graduate Aeronautical Laboratories, Pasadena, CA, AIAA Student Member

${ }^{\dagger}$ Assistant Professor, Graduate Aeronautical Laboratories, Pasadena, CA, AIAA Member
} 
a length of 4-5D, communication between the opposing separated shear layers could be prevented and large scale, organized vortex shedding completely suppressed, with an accompanying decrease in cylinder drag. Unal and Rockwell ${ }^{9,10}$ further investigated the wake formation region in the presence of a long plate placed a varying distance downstream of the cylinder, while Anderson and Szewczyk ${ }^{11}$ incorporated the effect of mild three-dimensionality, and Gerrard ${ }^{12}$ investigated the controlling effect of a plate that was normal to the flow.

In the case of the sphere, the mechanism by which obstructions affect the wake is less clear. On the one hand, a large sting may reduce cross-wake communication for the axisymmetric shear layer, but in order to do this the sting must present a large reduction in wake area. In this study, we seek to investigate the mechanism by which a sting affects the wake development.

\section{Experimental Setup}

The experiments were performed in the $\mathrm{NOAH}$ free surface water tunnel at the California Institute of Technology, which has a test section width of $0.46 \mathrm{~m}$, depth of $0.50 \mathrm{~m}$, and length of $1.6 \mathrm{~m}$. Uniform flow is achieved by employing honeycomb flow straighteners, turbulence suppressing screens, and a four-to-one fifth order polynomial contraction. ${ }^{13}$ The test section velocity range is between 0.03 and $0.40 \mathrm{~m} / \mathrm{s}$ and the sphere is centered in the test section, approximately $0.5 \mathrm{~m}$ downstream from the end of the contraction.

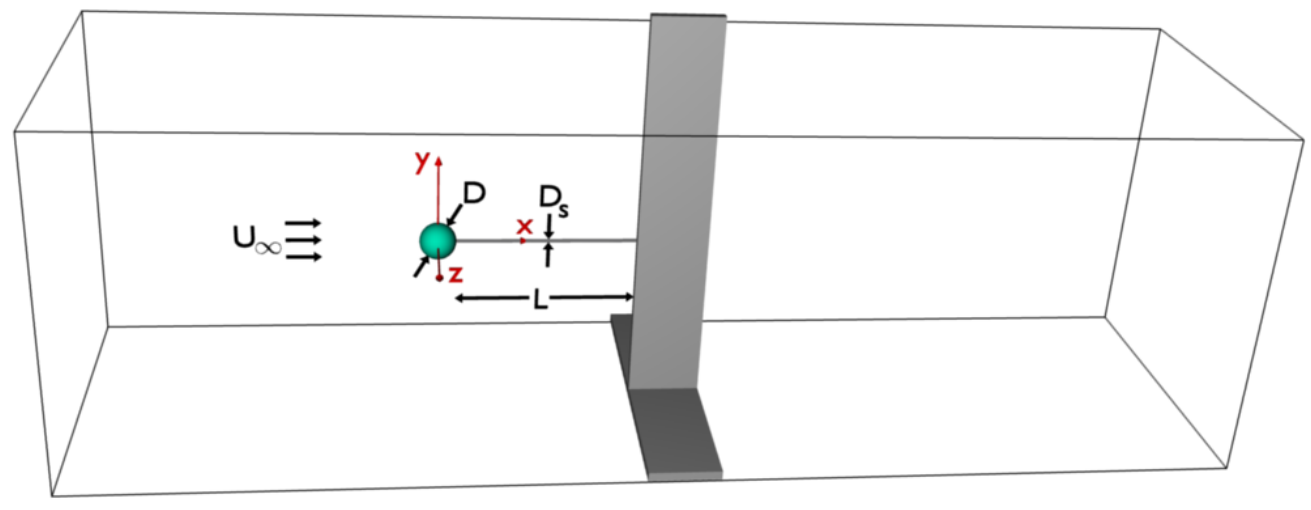

Figure 1: Experimental setup showing test section, notation, and coordinate system

The spheres were fabricated from polycarbonate, with a diameter tolerance of better than $\pm 0.0032 D$, and a sphericity of better than $0.0025 D$. The roughness height, $k$, is estimated to be less than $5 \mu \mathrm{m}$, or a maximum $k / D<2 \times 10^{-4}$, and the maximum area blockage ratio was less than $1 \%$. Both blockage and roughness are expected to have negligible effect on the sphere boundary layer and wake development at the Reynolds numbers being investigated, ${ }^{14}$ such that the influence of the sting size is effectively isolated.

The stings consisted of stainless steel rods with a straightness tolerance of better than $0.05 \%$, and a diameter tolerance of better than $-0.0016 D_{S}$. The minimum allowable rod diameter due to structural constraints was determined by estimating the angular misalignment of the sting to the oncoming flow due to deflections caused by gravity and unsteady fluid forcing using linear beam theory. The sting length was a minimum of eight times the diameter of the spheres under test (for comparison, Strouhal numbers in the wake of a cylinder were influenced by the splitter plate up to six diameters downstream ${ }^{8}$ ).

High speed particle image velocimetry (PIV) was used to investigate planar cuts of unsteady flow over an axisymmetric body. A LaVision system consisting of a pulsed ND-YLF laser, a high speed camera with a 1-megapixel CCD and 10 bit depth, and a computer with a precision timing unit was used. The laser sheet was split and directed to enter the test section from the top and bottom. The laser sheets were aligned to the flow to within $1^{\circ}$, and to each other within $0.3^{\circ}$. This allowed illumination of the entire field of view even with the opaque sting, which was necessary for structural stability. Saturation of the CCD due to reflections was avoided by spray-painting the spheres and stings black and carefully aligning the stings with the laser sheet.

The high frame rate elucidates the temporal evolution of the wake vortex dynamics, but it limits the amount of data that can be taken on the relatively slow large-scale wake shedding, due to memory limitations. 


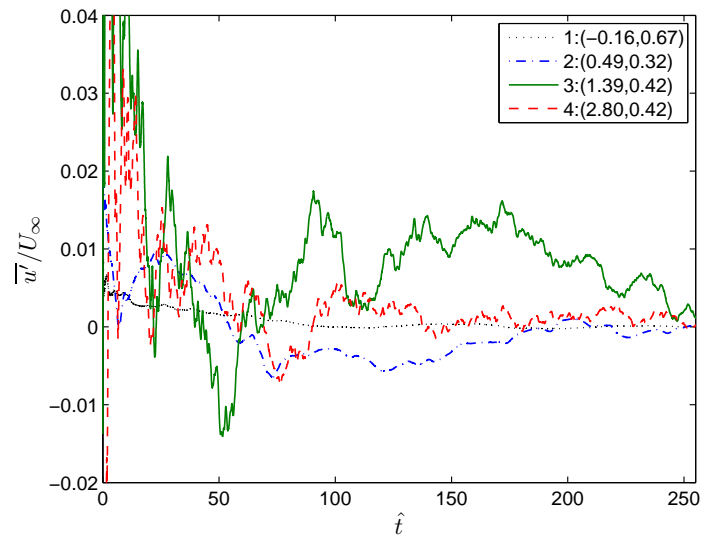

Figure 2: Convergence of the streamwise velocity (1)outside wake (2)behind sphere (3)near max $\overline{u^{\prime} u^{\prime}}$ and (4)downstream in wake, for $D=0.016 \mathrm{~m}$ and $\chi=0.50$, with position indicated by $(\mathrm{x} / \mathrm{D}, \mathrm{y} / \mathrm{D})$

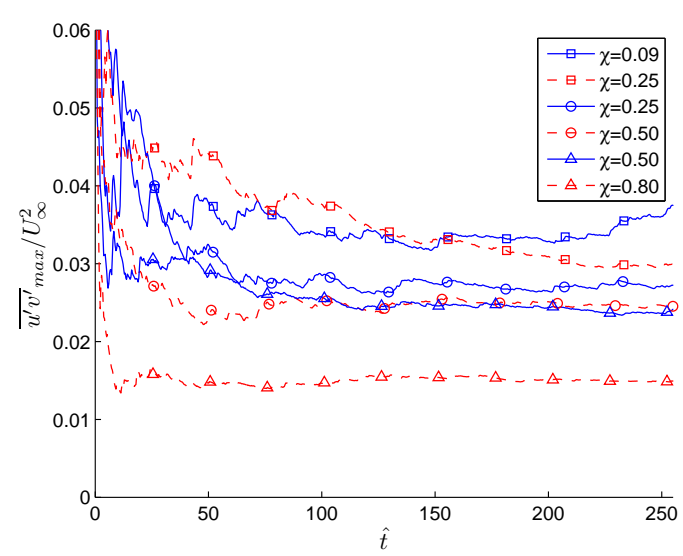

Figure 3: Convergence of the maximum Reynolds shear stress in the wake, $\overline{u^{\prime} v^{\prime}}{ }_{\max } / U_{\infty}^{2}$. See table 1 for symbols

In order to increase the total time of data collection, the laser pulse separation was increased as large as possible, to the point where further increase would lead to bad correlations due to out-of-plane velocity and a strong shear layer. This corresponds to a non-dimensional pulse separation of approximately $\Delta \hat{t}=\frac{1}{S t}=$ 0.016, which will vary depending on laser sheet thickness and post-processing techniques. In comparison, the timescales of interest for $R e=3800$ are approximately $\Delta \hat{t}=0.9$ for the shear layer instability and $\Delta \hat{t}=5$ for the wake instability. By minimizing the resolution of our images, we were able to collect continuous data for $\Delta \hat{t}=260$.

Table 1: Run conditions

\begin{tabular}{|l|l|l|c|}
\hline$D(m)$ & $U_{\infty}(m / s)$ & $\chi$ & symbol \\
\hline 0.051 & 0.070 & $0.09,0.13,0.16,0.19,0.25$ & $\square$ \\
\hline 0.026 & 0.139 & $0.25,0.31,0.39,0.50$ & $\bigcirc$ \\
\hline 0.016 & 0.223 & $0.50,0.60,0.80$ & $\triangle$ \\
\hline
\end{tabular}

The parameter space for this investigation included three different sphere sizes and five different sting sizes, which, taking into account the structural integrity of the stings, allowed for the range of $\chi$ shown in table 1. The free stream turbulence, $\sqrt{\overline{u^{\prime} u^{\prime}}} / U_{\infty}$, was measured using PIV and found to be $1.7 \%$ for the smaller two spheres and $2.6 \%$ for the larger sphere, where $u^{\prime}(t)=u(t)-\bar{u}$ is the fluctuating velocity at a point, and $\bar{u}=\frac{1}{N} \sum_{i=1}^{N} u_{i}$ is the mean at a point over all $\mathrm{N}$ samples. The larger free stream turbulence for the biggest sphere is likely due to flow unsteadiness at the low free stream velocity. The smallest sphere had low amplitude vibrations of less than $0.002 D$, due to slight vibrations of the supporting mount at the higher speed. This was determined from the time series of images, and no vibrations were detected for the larger spheres. Previous researchers have shown that free stream turbulence ${ }^{15}$ and acoustic excitation ${ }^{2}$ can alter the wake development, so it is not expected that the results for the different sphere sizes will completely collapse for a given $\chi$.

\section{Results}

\section{A. Convergence}

It has been suggested that the wake of a sphere oscillates in a streamwise-aligned plane that rotates slowly and randomly about the sphere center. ${ }^{16}$ Hence, in order to obtain converged statistics using PIV, a significant 


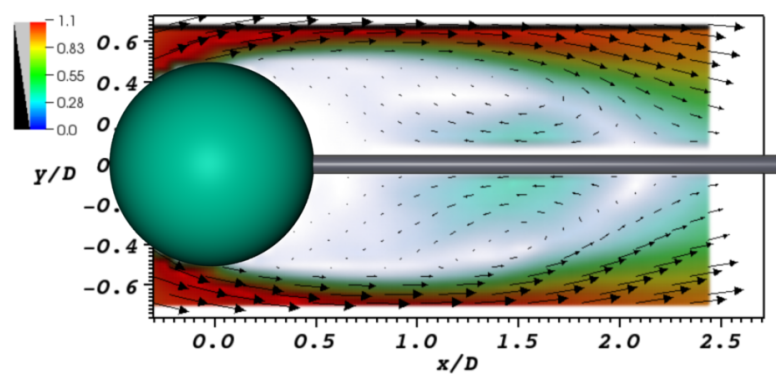

(a)

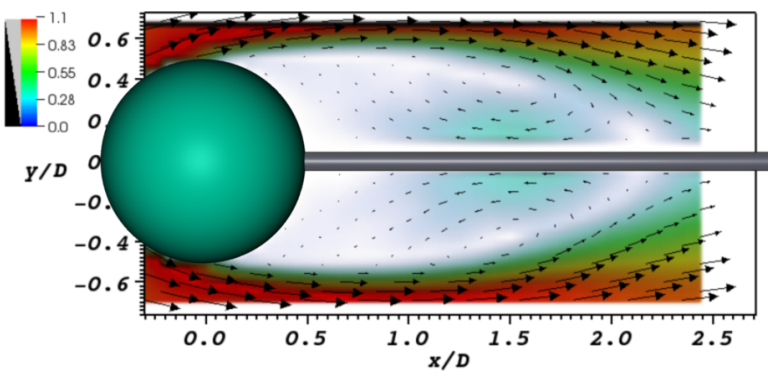

(b)

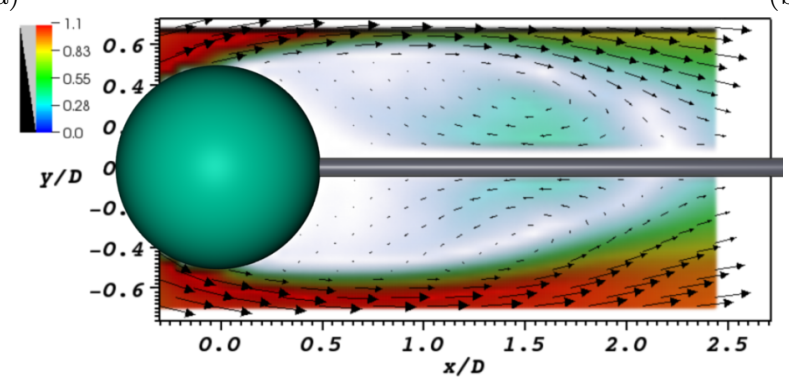

(c)

Figure 4: Consecutive averages over $\hat{t}=50$ showing large scale movement of the wake for $\chi=0.09$, with color indicating non-dimensional velocity magnitude (a) $100<\hat{t}<150$ (b) $150<\hat{t}<200$ (c) $200<\hat{t}<250$

number of wake oscillations must be averaged over such that the variations in the averaged wake quantities due to the random orientation of the plane tend toward a constant value. As shown in figure 2, in areas of low turbulence, such as the outer flow and right behind the sphere, convergence is obtained rapidly, whereas in areas of high turbulence there are still significant fluctuations even though averages were taken over approximately 50 wake oscillations. Though it is difficult to determine using just a planar cut, it appears that there is a movement of the wake with a frequency lower than that of the wake instability. This was confirmed by examining the temporal data for $\chi=0.09$, which revealed that the orientation of the entire wake seems to move slowly. Figure 4 shows three consecutive averages over a dimensionless time of $\hat{t}=50$ ( $\sim 10$ wake oscillations), in which the mean wake is seen to move from being oriented slightly below the sting to significantly above it. It would not have been obvious that the entire wake was moving if only half of the plane had been imaged. This movement was seen to varying degrees for the different $\chi$, likely because we are only examining a planar cut, and motion normal to the field of view would not be easily detected. The relation of the large-scale wake motion and the wake instability warrants further investigation. A low frequency motion was also found in the computational study of Yun et al. ${ }^{5}$ in the analysis of drag and lift. For $R e=3700$, they found the frequency of the large scale lift motion to be $S t \approx 0.025(\Delta \hat{t} \approx 40)$. This makes it difficult to obtain converged data, especially if attempting to simultaneously resolve the high frequency shear roll-up. Not shown here, we found that at $R e=12,000$ convergence took even longer, as indicated by Yun et al. ${ }^{5}$ for $R e=10,000$.

Figure 3 shows the convergence of the maximum Reynolds shear stress (average of maximum from the top and bottom wake), $\overline{u^{\prime} v^{\prime}}$ max $/ U_{\infty}^{2}$, for a representative sample of different $\chi$. A similar trend is seen for $\overline{v^{\prime} v^{\prime}}$, but convergence is worse for $\overline{u^{\prime} u^{\prime}}$ due to the large-scale motion of the wake in the radial direction, and the steep gradient of the streamwise velocity in the shear layer.

\section{B. Mean Wake}

As observed by previous researchers, ${ }^{2,15}$ we have also found that the length of the mean recirculation region reduces due to both higher free stream turbulence and disturbances near the natural frequency of the shear layer instability. Figure 5 shows the average velocity field overlaid with the dimensionless Reynolds shear stress. In figures $5 \mathrm{a}$ and $5 \mathrm{~b}$ the same $\chi$ is shown, but the free stream turbulence is higher in the former case, which causes a more rapid growth of the shear layer instability, leading to a shorter wake length, which is here defined as the location along the sting at which the streamwise velocity changes directions (which 


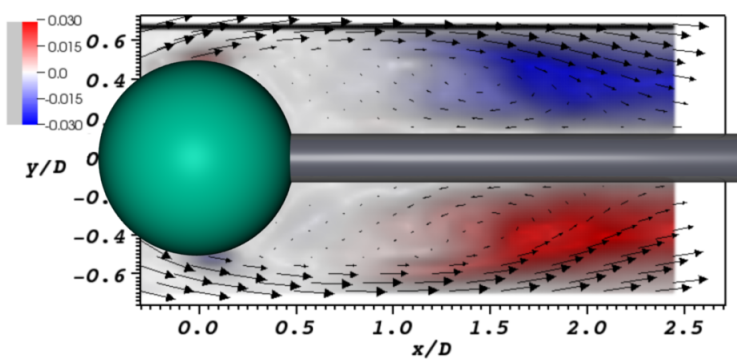

(a)

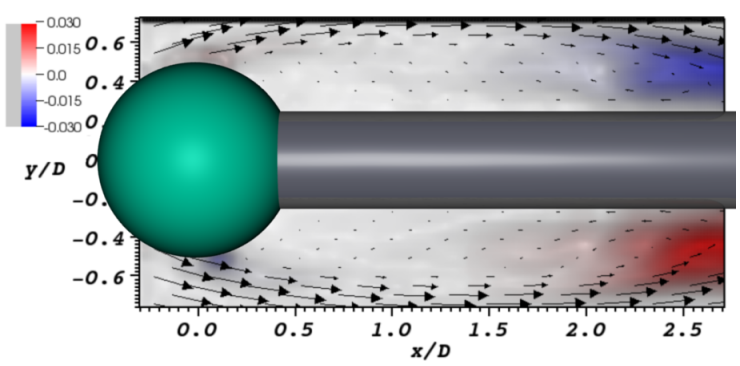

(c)

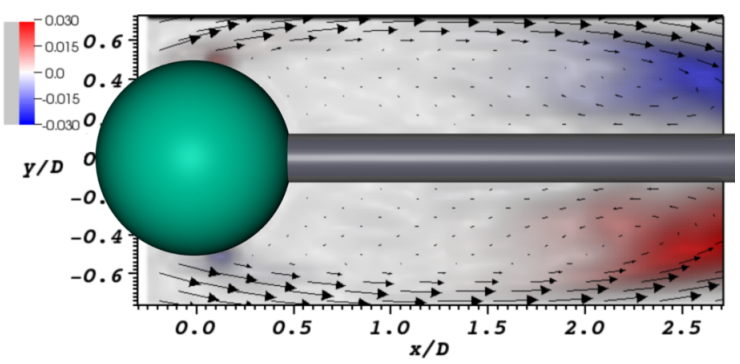

(b)

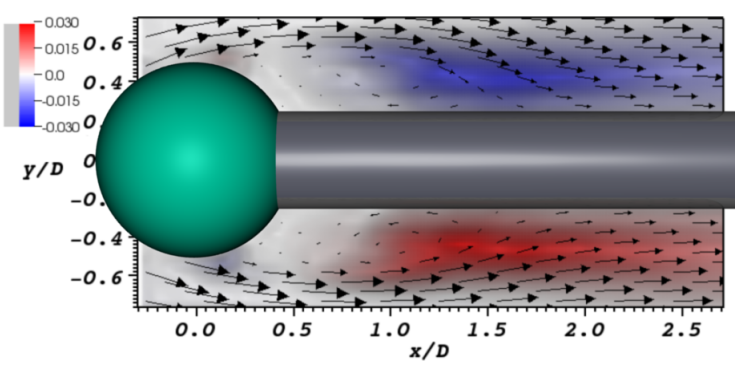

(d)

Figure 5: Mean velocity field overlaid with the mean Reynolds shear stresses $\overline{u^{\prime} v^{\prime}} / U_{\infty}^{2}(\mathrm{a}, \mathrm{b}) \chi=0.25$ : left has higher free stream turbulence $(\mathrm{c}, \mathrm{d}) \chi=0.50$ : right sphere has low amplitude vibrations

could be considered a mean stagnation point). In Figures $5 \mathrm{c}$ and $5 \mathrm{~d}$ a larger $\chi$ is shown, with the difference between the two being that the latter has disturbances in the boundary conditions which have a frequency peak that lies within the $S t$ range measured by others for the shear layer instability, as will be detailed in subsection C.

Even with these differences in wake length, it is still possible to examine the effect of $\chi$ by comparing the $\chi$ for each sphere size. To examine the shape of the average velocity magnitude, a cubic spline curve was fit to each column of data to find the position of the maximum and minimum velocity to subpixel accuracy. The velocity contour $u=U_{\infty} / 2$, was also determined as it is useful for comparing the mean wake shape for the different sphere sizes. Here we will focus on the smallest sphere (largest $\chi$ ), as no differences were found for the smaller $\chi$, within the scatter of the data. Figure 6a shows representative paths of the mean wake for the three different $\chi$ of the smallest sphere. Within the scatter of the data, the mean path of the wake does not change with $\chi$, except for perhaps between a $\chi$ of 0.6 and 0.8 . This indicates that the mean path of the wake is largely determined by the energetic outer flow. Along these minimum and maximum paths, the velocity magnitude is similar for the different $\chi$, up to approximately $\chi=0.50$ (not shown). For larger $\chi$ (figure 6b) there are deviations in the downstream maximum, and significant changes in the shape of the minimum, indicating a change in how fluid is transferred to and from the mean recirculation region. However, more data is necessary to determine how the wake length and $\chi$ interact, as it is possible that $\chi<0.50$ also produce deviations in the mean velocity magnitude for the case of the short wake.

To compare the effect of free stream turbulence and vibrations, the $U_{\infty} / 2$ contour is shown in figure 7 for all $\chi$. This demonstrates that the importance of $\chi$ on the path of the mean wake is almost insignificant compared with these other effects. This trend would need to be examined for different flow regimes, such as higher Reynolds numbers where the shear layer transitions further upstream ${ }^{5}$ and the Strouhal number of the shear layer becomes much larger than that of the wake instability. ${ }^{1}$

An example of the mean Reynolds stress fields are shown in figures 8 and $5 \mathrm{~d}$, for the small sphere with $\chi=0.50$. The average of the top and bottom maximum of the Reynolds stresses are shown in figure 9, along with those obtained in the computational study of Yun et al. ${ }^{5}$ (for a Reynolds number of 3700). The data is noisy for smaller $\chi$ due to convergence issues, but a general trend of decreasing stresses with increasing $\chi$ is observed. The error bars are meant to give a sense of the convergence, and indicate the maximum and 


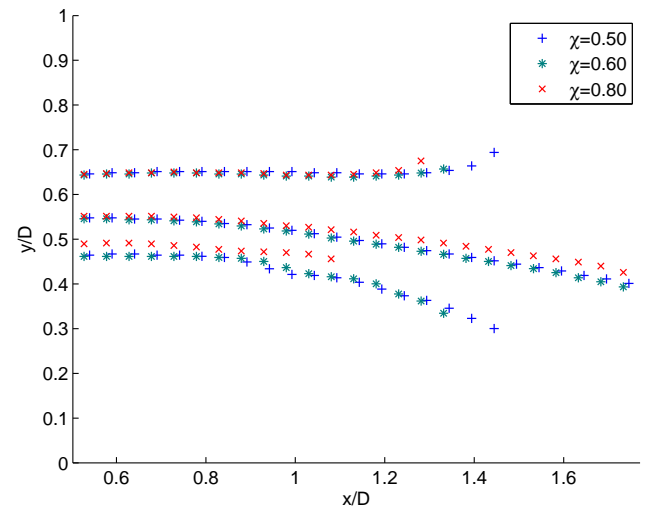

(a)

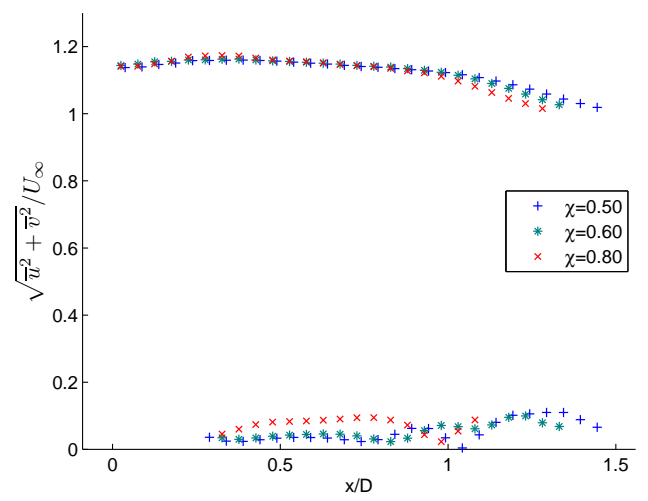

(b)

Figure 6: Shape of the mean wake for $D=0.016 \mathrm{~m}$ : (a) paths of maximum (top), minimum (bottom), and constant $U_{\infty} / 2$ (center) velocity magnitude. (b) Velocity magnitude along the maximum and minimum paths

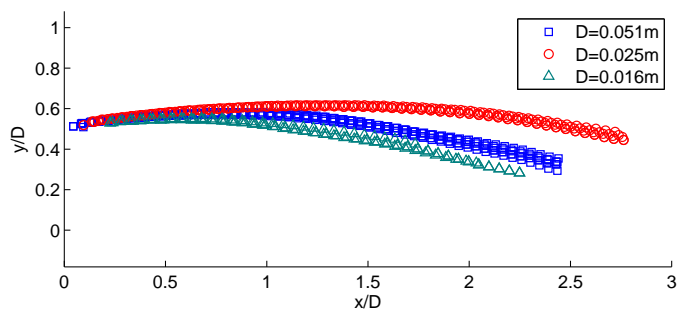

Figure 7: Paths of constant $U_{\infty} / 2$ for each sphere size, showing influence of free stream turbulence and vibrations

minimum values from the convergence plots (see, e.g. figure 3 ) for $\hat{t}>125$. Particularly noteworthy is the rapid drop in $\overline{v^{\prime} v^{\prime}}$ between $\chi=0.60$ and 0.80 , because the sting is growing in the $y$-direction. Here the area blockage ratio, $\chi^{2}$, jumps from 0.36 to 0.64 . It would be useful to fill in $\chi$ around 0.80 to verify and better understand this trend. It should also be noted that, within the scatter of the data, the higher free stream turbulence and the vibrations simply caused an earlier growth of the shear instability and did not significantly affect the maximum Reynolds stresses.

\section{Periodic behavior}

Strong frequency peaks corresponding to the wake instability were not detected in this study, possibly because the magnitude of the oscillations are small in the near wake, and also the Reynolds number of the current experiments lies in the range in which Sakamoto and Haniu ${ }^{1}$ labeled a transitional region because no distinct frequency peaks were detected. In addition, no frequency peaks corresponding to the shear layer instability were found for the two sphere sizes which had longer wakes, likely due to the slow roll-up of the shear layer. However, for the short wake the shear instability was detected easily by taking a fast Fourier transform of the time-resolved PIV vector fields.

Figure 10 shows a map of the wake with color indicating the strength of the shear layer peak, normalized by the maximum value in each image. The left side shows the streamwise $(u)$ component, while the right shows the radial $(v)$ component. These patterns are to be expected for vortices rolling up along a shear layer.

Comparing the top $(\chi=0.50)$ and bottom $(\chi=0.80)$ parts of figure 10 , it is seen that the larger sting 


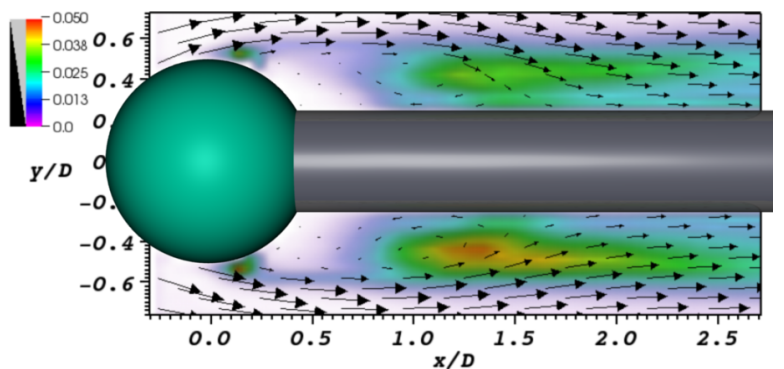

(a)

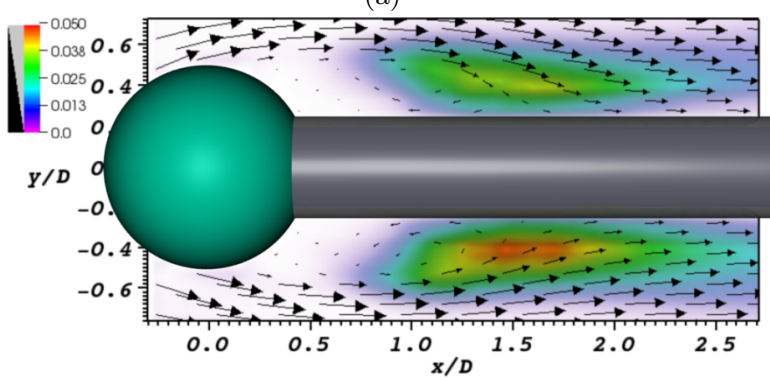

(b)

Figure 8: Mean Reynolds stresses for $\chi=0.50$, $D=0.016 \mathrm{~m}(\mathrm{a}) \overline{u^{\prime} u^{\prime}}(\mathrm{b}) \overline{v^{\prime} v^{\prime}}$

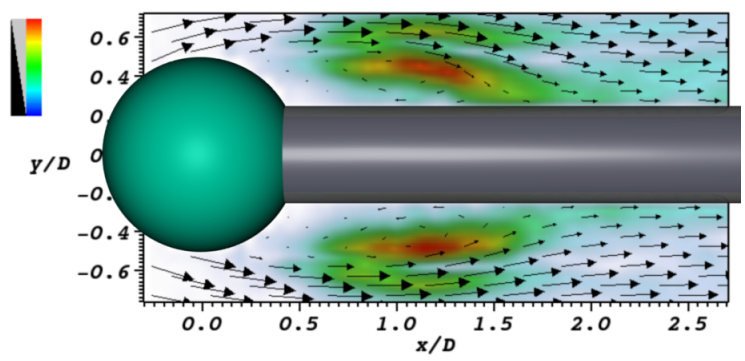

(a)

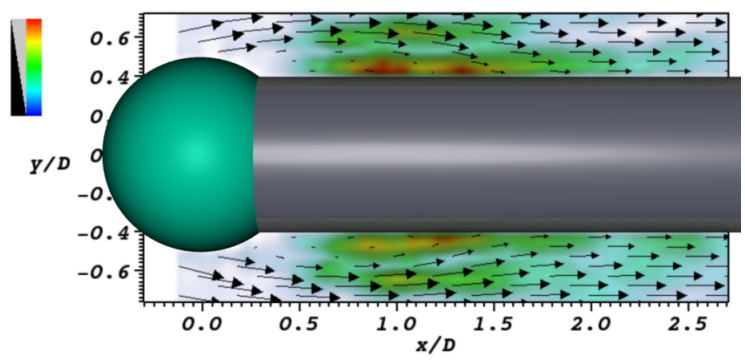

(c)

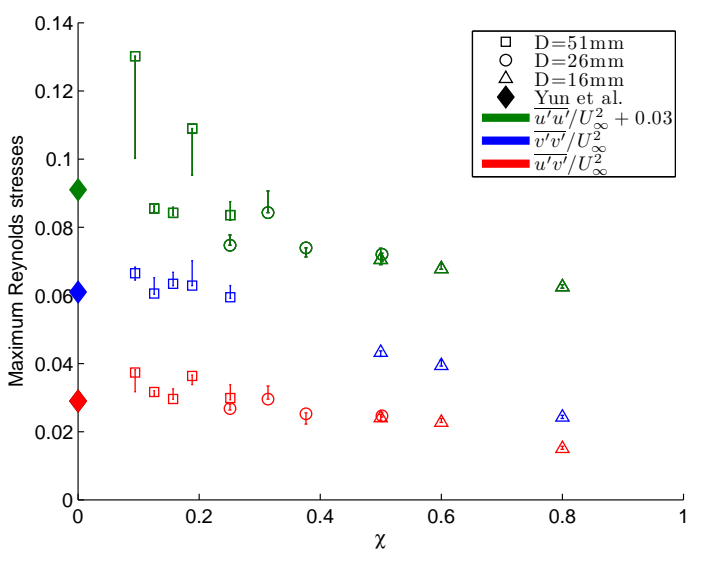

Figure 9: Maximum of mean Reynolds stresses as a function of $\chi$, with error bars based on convergence. Computational results from Yun et al. (Ref. 5) shown for comparison

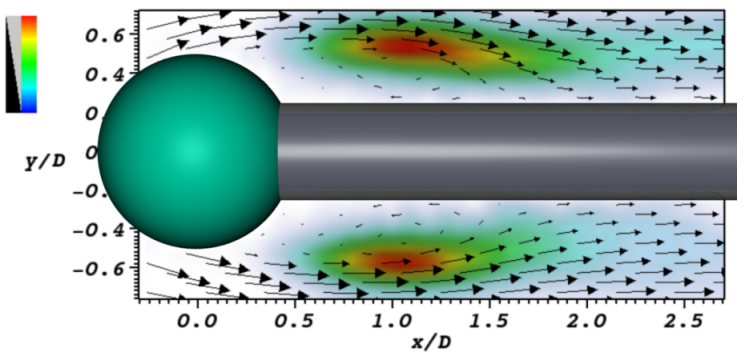

(b)

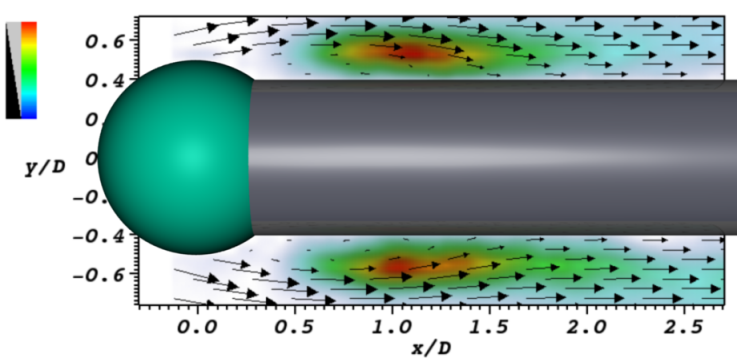

(d)

Figure 10: Magnitude of frequency spectrum peak (streamwise on the left and radial on the right) as a function of position, normalized by the maximum value for each image, where the maximum peak in (c) is $65 \%$ as strong as in (a), and the maximum peak in (d) is $58 \%$ as strong as in (b). (a,b) $\chi=0.50, S t=1.085$ $(\mathrm{c}, \mathrm{d}) \chi=0.80, S t=1.081$ 
significantly encroaches on the vortex paths of the smaller sting case. Also, the maximum peak for the larger sting is $65 \%$ as strong as that of the smaller sting for the $u$-component and $58 \%$ for the $v$-component (whereas the strength of the maximum peaks for $\chi=0.60$ is diminished by less than $10 \%$ ). Thus the roll-up of the shear layer is damped, which matches well with the Reynolds stresses decreasing with increasing $\chi$.

As shown in figure 11, the peaks of the shear layer frequency in the wake agree well with the low amplitude frequency of the sphere vibrations. It appears that the facility noise, in the form of acoustic excitation and/or sphere vibrations, enhanced the instability of the shear layer. For comparison, Sakamoto and Haniu ${ }^{1}$ found the frequency of shear layer roll-up to range from $1.0<S t<1.3$ for Re near 3800. For $\chi=0.80$ both the vibration peak and the wake peak are slightly broader.

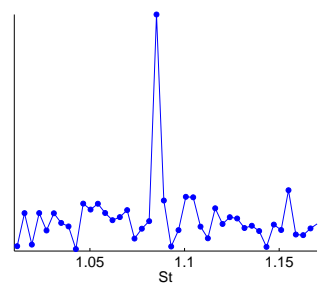

(a)

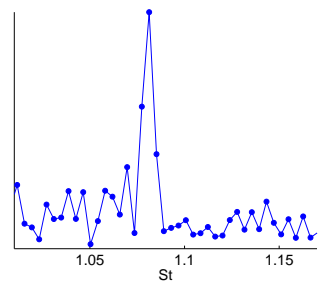

(c)

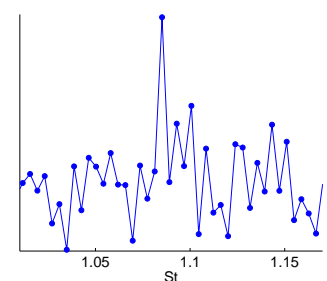

(b)

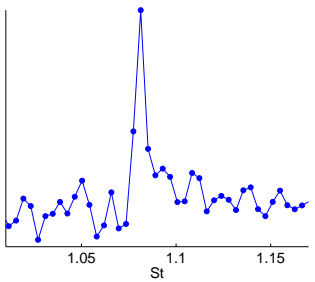

(d)

Figure 11: (a,c) Representative frequency spectrum of u-velocity and (b,d) vibration frequency of spheres, for $\chi=0.50$ on the top and $\chi=0.80$ on the bottom

\section{Conclusion}

We have performed preliminary investigations into the effect of the sting size on flow over a sphere using high speed PIV. Due to the low frequency wake oscillations, and an apparent lower frequency wake motion, data needs to be taken over many wake oscillations to achieve convergence. Here we integrated over a nondimensional time of 250, which was sufficient to draw general conclusions. However, acquiring data over a longer time would be preferred. It was also noted that it appears to be more difficult to obtain converged statistics at higher subcritical Reynolds numbers.

As expected, the effect of a a sting behind a sphere is a weak analogy to the splitter plate behind a cylinder, due to the three-dimensional nature of the sting.

The mean wake was shown to not change significantly for $\chi<0.50$, and the Reynolds stresses decrease with increasing $\chi$. It was also shown that both increased free stream turbulence and excitation at a naturally unstable frequency have a larger impact on the shape of the mean wake than $\chi$, at least for $\chi<0.80$.

Disturbing the flow at a frequency within the range of naturally unstable shear layer frequencies was shown to produce early transition of the shear layer, causing the vortices to roll-up at the same frequency as the acoustic noise and/or sphere vibrations. The energy associated with this frequency drops dramatically when $\chi$ is increased from 0.60 to 0.80 .

Thus, taking into account the shape of the mean wake, the maximum mean Reynolds stresses, and the shear layer instability, it appears that even with $\chi=0.50$ the properties of the mean wake are only slightly changed from that of a sphere with a negligible diameter support. Of course, it would be best to use the smallest sting possible, however, using a $\chi$ up to $\sim 0.25$ seems appropriate if needed. This would need to be verified for other Reynolds numbers, and the effect on the wake instability should also be investigated. 
These conclusions may change if the interest lies in examining unsteady forces or instantaneous fields.

\section{Acknowledgments}

Support from the National Science Foundation under Grant No. 0747672 (Program Manager William W. Schultz) is gratefully acknowledged.

\section{References}

\footnotetext{
${ }^{1}$ Sakamoto, H. and Haniu, H., "A study on vortex shedding from spheres in a uniform flow," ASME, Transactions, Journal of Fluids Engineering, Vol. 112, 1990, pp. 386-392.

${ }^{2} \mathrm{Kim}, \mathrm{H}$. and Durbin, P., "Observations of the frequencies in a sphere wake and of drag increase by acoustic excitation," Physics of Fluids, Vol. 31, 1988, pp. 3260.

${ }^{3}$ Bakić, V. and Perić, M., "Visualization of flow around sphere for Reynolds numbers between 22000 and 400000 ," Thermophysics and Aeromechanics, Vol. 12, No. 3, 2005, pp. 307-315.

${ }^{4}$ Achenbach, E., "Vortex shedding from spheres," Journal of Fluid Mechanics, Vol. 62, No. 02, 1974, pp. $209-221$.

${ }^{5}$ Yun, G., Kim, D., and Choi, H., "Vortical structures behind a sphere at subcritical Reynolds numbers," Physics of Fluids, Vol. 18, 2006, pp. 015102.

${ }^{6}$ Constantinescu, G. and Squires, K., "LES and DES Investigations of Turbulent Flow over a Sphere at Re= 10,000," Flow, Turbulence and Combustion, Vol. 70, No. 1, 2003, pp. 267-298.

${ }^{7}$ Hoerner, S., "Tests of Spheres With Reference to Reynolds Number, Turbulence, and Surface Roughness. Technical Memoradum No. 777," National Advisory Committee for Aeronautics, Langley, Virginia, 1935.

${ }^{8}$ Roshko, A., "On the wake and drag of bluff bodies," J. Aeronaut. Sci, 1955.

${ }^{9}$ Unal, M. and Rockwell, D., "On vortex formation from a cylinder. Part 1. The initial instability," Journal of Fluid Mechanics Digital Archive, Vol. 190, 1988, pp. 491-512.

${ }^{10}$ Unal, M. and Rockwell, D., "On vortex formation from a cylinder. Part 2. Control by splitter-plate interference," Journal of Fluid Mechanics Digital Archive, Vol. 190, 1988, pp. 513-529.

${ }^{11}$ Anderson, E. and Szewczyk, A., "Effects of a splitter plate on the near wake of a circular cylinder in 2 and 3-dimensional flow configurations," Experiments in Fluids, Vol. 23, No. 2, 1997, pp. 161-174.

${ }^{12}$ Gerrard, J., "The mechanics of the formation region of vortices behind bluff bodies," Journal of Fluid Mechanics, Vol. 25, No. 02, 1966, pp. 401-413.

${ }^{13}$ Gharib, M., The effect of flow oscillations on cavity drag, and a technique for their control, Ph.D. thesis, California Institute of Technology, 1983.

${ }^{14}$ Achenbach, E., "The effects of surface roughness and tunnel blockage on the flow past spheres," Journal of Fluid Mechanics, Vol. 65, No. 01, 1974, pp. 113-125.

${ }^{15}$ Bakic, V., "Experimental Investigation of a Flow Around a Sphere," Thermal Science, Vol. 8, 2004, pp. 63-81.

${ }^{16}$ Taneda, S., "Visual observations of the flow past a sphere at Reynolds numbers between $10^{4}$ and $10^{6}$," Journal of Fluid Mechanics, Vol. 85, No. 01, 1978, pp. 187-192.
} 